\title{
In-Hospital Postoperative Mortality Rates for Selected Procedures in Tanzania's Lake Zone
}

\author{
Taylor Wurdeman $^{1,2}$ (1) $\cdot$ Christopher Strader $^{1,3} \cdot$ Shehnaz Alidina $^{1} \cdot$ David Barash $^{4} \cdot$ Isabelle Citron $^{1}$. \\ Ntuli Kapologwe $^{5}$ - Erastus Maina ${ }^{6}$ - Fabian Massaga ${ }^{7}$ Adelina Mazhiqi ${ }^{1,8} \cdot$ John G. Meara ${ }^{1,9}$. \\ Gopal Menon $^{1} \cdot$ Cheri Reynolds $^{10} \cdot$ Meaghan Sydlowski $^{1} \cdot$ John Varallo $^{11} \cdot$ Sarah Maongezi $^{12}$. \\ Mpoki Ulisubisya ${ }^{12}$
}

\begin{abstract}
Background Postoperative mortality rate is one of six surgical indicators identified by the Lancet Commission on Global Surgery for monitoring access to high-quality surgical care. The primary aim of this study was to measure the postoperative mortality rate in Tanzania's Lake Zone to provide a baseline for surgical strengthening efforts. The secondary aim was to measure the effect of Safe Surgery 2020, a multi-component intervention to improve surgical quality, on postoperative mortality after 10 months.

Methods We prospectively collected data on postoperative mortality from 20 health centers, district hospitals, and regional hospitals in Tanzania's Lake Zone over two time periods: pre-intervention (February to April 2018) and post-intervention (March to May 2019). We analyzed postoperative mortality rates by procedure type. We used logistic regression to determine the impact of Safe Surgery 2020 on postoperative mortality.

Results The overall average in-hospital non-obstetric postoperative mortality rate for all surgery procedures was $2.62 \%$. The postoperative mortality rates for laparotomy were $3.92 \%$ and for cesarean delivery was $0.24 \%$. Logistic regression demonstrated no difference in the postoperative mortality rate after the Safe Surgery 2020 intervention. Conclusions Our results inform national surgical planning in Tanzania by providing a sub-national baseline estimate of postoperative mortality rates for multiple surgical procedures and serve as a basis from which to measure the impact of future surgical quality interventions. Our study showed no improvement in postoperative mortality after implementation of Safe Surgery 2020, possibly due to low power to detect change.
\end{abstract}

Taylor Wurdeman and Christopher Strader are Co-First author.

Sarah Maongezi and Mpoki Ulisubisya are Co-Senior author.

Electronic supplementary material The online version of this article (https://doi.org/10.1007/s00268-020-05802-w) contains supplementary material, which is available to authorized users.

Taylor Wurdeman

Taylor.wurdeman@gmail.com

1 Program in Global Surgery and Social Change, Harvard Medical School, 641 Huntington Avenue, Boston, MA 02115, USA

2 University of Miami Miller School of Medicine, Miami, FL, USA

\section{Introduction}

Surgery is an integral part of effective health systems, with surgical disease accounting for $30 \%$ of the global burden of disease [1]. The Lancet Commission on Global Surgery

3 Department of Surgery, University of Massachusetts, Worcester, MA, USA

4 GE Foundation, Boston, MA, USA

5 Department of Health, Social Welfare and Nutrition Service, President's Office - Regional Administration and Local Government, Dodoma, Tanzania

6 Dalberg Advisors, New York, NY, USA 
(LCoGS) identified that 5 billion people lack access to safe, timely, affordable surgical and anesthesia care [2]. Lowand middle-income countries (LMICs) bear the majority of the burden of surgical disease [3]. If surgical care was scaled up in LMICs, 1.4 million deaths could be averted annually [3]. Scaling surgical care in LMICs requires a focus on maternal surgical care, as cesarean section is one of the most common surgical procedures in LMICs [4] and is a necessity for maternal/neonatal survival in $19 \%$ of all deliveries [5]. LMICs need to perform 143 million additional surgical procedures per year in order to match the surgical burden of disease [2]. Reaching these goals requires augmentation of surgical provider workforce, scalable technology, improved data quality, and financial access to surgery.

While scaling surgical capacity is important, efforts must also address surgical quality. The postoperative mortality rate (POMR) of elective surgeries in Africa is twice the global average [6]. Furthermore, a recent study found that African women are 50 times more likely than non-African women to die following a cesarean delivery [7]. Surgical and anesthesia systems without quality and safety lead to increased rates of surgical complications, including death [8-10]. While quality is difficult to codify, POMR has been identified as an important indicator encompassing multiple surgical complications. POMR is one of six surgical indicators identified by LCoGS for monitoring access to high-quality surgical care [2]. POMR highlights systems with poor surgical quality or late disease presentation due to poor access to care and gives a starting point to measure precipitating factors. While POMR has been identified as an important indicator, difficulties in measurement have led to few reliable national estimates. The 2016 report on Surgical World Development Indicators showed only 29 countries reporting on perioperative mortality [11]. Historically, POMR has been difficult to track consistently in LMICs, partly due to lack of robust health information systems in many LMICs. Very few studies in Africa report POMR [6, 12]. One difficulty in generating national POMR estimates is the heterogeneity

7 Bugando Medical Centre, Consultant and Teaching University Hospital, Mwanza, Tanzania

8 Department of Internal Medicine, Ängelholm Hospital, Ängelholm, Sweden

9 Department of Plastic and Oral Surgery, Boston Children's Hospital, Boston, MA, USA

10 Assist International, Ripon, CA, USA

11 Jhpiego, Baltimore, MD, USA

12 Ministry of Health, Community Development, Gender, Elderly \& Children, Dodoma, Tanzania of definitions. The World Health Organization (WHO) and the LCoGS define POMR as death before discharge from the hospital or within 30 days, whichever is sooner [13]. Despite the agreed definition, some studies have included deaths outside of the study hospital. Crude measurements of POMR include every surgical procedure in the denominator, making them sensitive to differences in complexity of cases between hospitals. Procedure-specific POMR may provide a more valid quality measure by capturing in the denominator only cases where there is a true risk of death. This study defines POMR as deaths before discharge or within 30 days, starting the moment the patient leaves the operating room.

Baseline measurement of POMR, while influenced by future changes in case numbers or case mix, is nonetheless a valid measure to monitor changes in quality over time. This paper combines prospective data collection with data from hospital medical records to estimate inpatient POMR for multiple procedure categories in 20 facilities in Tanzania's Lake Zone. Our primary aim of this study is to provide baseline measurements of POMR in Tanzania's Lake Zone. Our secondary aim is to evaluate the effect of the Safe Surgery 2020 (SS2020) intervention on POMR after one year.

\section{Materials and methods}

\section{Study design}

This prospective, longitudinal study of POMR was designed to provide a baseline measurement of POMR in Tanzania's Lake Zone and to assess the impact of the SS2020 intervention on in-hospital POMR.

\section{Study setting and participants}

This study took place at 20 facilities in Tanzania's Lake Zone including health centers, district hospitals, and regional hospitals (Table 1). The facilities were divided into two groups based on their geographical location (Fig. 1). Surgical inpatients including cesarean delivery were included. Vaginal deliveries were also followed to provide comparison data.

\section{Intervention}

The SS2020 multicomponent intervention was implemented over 10 months to improve surgical quality. The first phase involved training surgical team members on leadership, teamwork, communication, and quality improvement techniques. The second phase involved training on the WHO's Surgical Safety Checklist (SSC), 
peri-operative infection prevention, safe cesarean delivery, sterilization protocols, data quality, and surgical techniques. The third phase involved bimonthly mentorship visits, virtual mentorship through Project ECHO, infrastructure grants up to $\$ 10,000$ USD, an equipment package, and the Touch Surgery mobile training application. A more detailed description of the intervention is described elsewhere [14].

\section{Data collection}

Tanzanian medical doctors were trained to collect data for the study, with each facility having at least one dedicated full-time data collector. Data were collected over two time periods: pre-intervention (February-April 2018) and postintervention (March-May 2019). All elective and emergency surgeries were observed on weekdays, and all emergencies at the weekends. Patients were evaluated starting postoperative day one for surgical procedures and from day zero for spontaneous vaginal deliveries. Data collectors made daily rounds to each surgical and postpartum inpatients and for up to 30 days or discharge, documenting the patient's mortality status. For this study, data were collected on demographics, surgery type, and time until death, where applicable. All data were collected using paper forms and transferred to REDCap daily.

\section{Analysis}

We define postoperative mortality as any death following a surgical or obstetric procedure within 30 days of the procedure or until discharge, whichever is sooner. Surgery patients discharged before 30 days who subsequently died at home or another hospital were not followed. Intra- operative deaths were also not included due to limitations in data collector availability, with observation of patients starting when they arrived to the postoperative ward. The denominator for the POMR calculation was the number of major surgeries, defined as a procedure requiring general/ regional anesthesia. POMR was stratified based on surgical category. Due to the relatively small number of deaths, three categories were used: cesarean delivery, laparotomy, and surgeries (excluding laparotomy) (see Table 2 footnotes for a full list). The definition of laparotomy for this study was a midline incision of the abdomen of varying lengths, which is more broad as compared with the Western definition of laparotomy. Examples in our data collection included classical laparotomy, total abdominal hysterectomy, and splenectomy. Procedures listed as laparotomy, but involving a horizontal incision as determined by clinical experience of an HIC surgeon, were excluded. To analyze the effect of the intervention on postoperative mortality, a logistic regression model was used with an interaction term between intervention status and time point. Unless explicitly stated, all results are presented as aggregates across control and intervention sites.

\section{Power calculation}

The study is predicted to be powered to $0.33-0.72$ for changes in non-obstetric POMR and 0.22 for cesarean delivery specific POMR before and after the SS2020 intervention. To adequately power the question of whether or not the SS2020 intervention had an effect on POMR would have required 797 non-obstetric surgeries and 7985 cesarean deliveries in the pre-intervention and post-intervention arms. Reaching these case numbers was not feasible within the larger SS2020 effort due to time and

Table 1 Facility characteristics are presented to show similarity of pre-intervention characteristics between control and intervention sites

\begin{tabular}{llll}
\hline Characteristics & $\begin{array}{l}\text { All facilities } \\
(n=20) n(\%)\end{array}$ & $\begin{array}{l}\text { Intervention facilities } \\
(n=10) n(\%)\end{array}$ & $\begin{array}{l}\text { Control facilities } \\
(n=10) n(\%)\end{array}$ \\
\hline $\begin{array}{l}\text { Level of facility } \\
\text { Health centre }\end{array}$ & $4(20 \%)$ & $2(20 \%)$ & $2(20 \%)$ \\
District hospitals & $11(55 \%)$ & $6(60 \%)$ & $5(50 \%)$ \\
Regional referral hospital & $5(25 \%)$ & $2(20 \%)$ & $3(30 \%)$ \\
Number of inpatient beds & & $3(30 \%)$ & $2(20 \%)$ \\
$0-100$ & $5(25 \%)$ & $6(60 \%)$ & $7(70 \%)$ \\
$101-300$ & $13(65 \%)$ & $1(10 \%)$ & $1(10 \%)$ \\
$300+$ & $2(10 \%)$ & 52 & 50 \\
Average number of major surgeries per facility & 102 & 1.6 & 258 \\
Number of functioning major ORs per facility & 1.7 & 330 & 1.7 \\
Average monthly inpatient volume per facility & 589 & &
\end{tabular}

Multiple hospital classifications and sizes were used for this study 


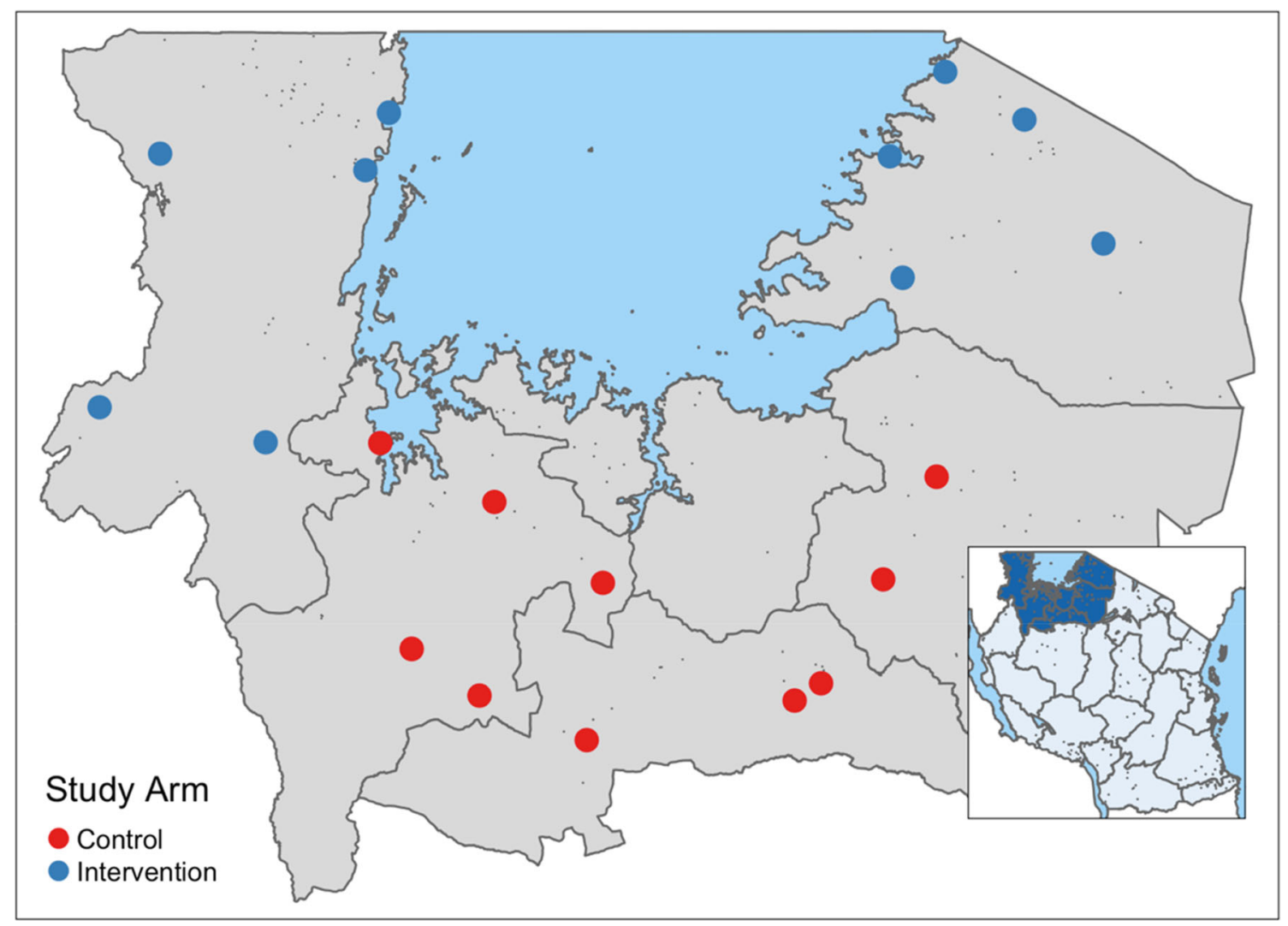

Fig. 1 Map of study sites in the Lake Zone of Tanzania

resource constraints. A full evaluation protocol of the SS2020 project can be read in Alidina et al. 2019 [14]. The details of assumptions for the power calculations can be found in Online Resource 1.

\section{Results}

The mean age of patients was 29.3 years. Females comprised $89.7 \%$ of the study population. The mean age of patients who required non-obstetric surgical procedures was 40.1 years. Table 3 describes the patient characteristics. Laparotomies accounted for $48.0 \%$ of non-obstetric procedures. Bellwether procedures, defined by LCoGS as the three procedures (cesarean delivery, laparotomy, and treatment of open fracture) that any well-equipped surgical center should provide, made up $86.2 \%$ of procedures. The majority $(85.4 \%)$ of the bellwether procedures conducted were cesarean deliveries.

Among the 6,158 patients followed across the intervention and control sites, there were 53 deaths. The inhospital non-obstetric POMR was $2.62 \%$. The laparotomy specific POMR was $3.92 \%$. Cesarean delivery POMR was lower than non-obstetric surgery POMR at $0.24 \%$. Cesarean delivery mortality was 8 times higher than spontaneous vaginal delivery mortality $(0.24 \%$ vs. $0.03 \%)$. All of the

Table 2 Aggregated postoperative mortality rate by procedure type

\begin{tabular}{llllll}
\hline Cohort & $\begin{array}{l}\text { Number } \\
\text { of deaths }\end{array}$ & $\begin{array}{l}\text { Number of } \\
\text { procedures }\end{array}$ & $\begin{array}{l}\text { Postoperative/CS } \\
\text { mortality rate }\end{array}$ & $\begin{array}{l}\text { Mean length of } \\
\text { stay in days, dead (sd) }\end{array}$ & $\begin{array}{l}\text { Mean length of } \\
\text { stay, alive (sd) }\end{array}$ \\
\hline Only laparotomies $^{\prime}$ & 30 & 765 & $3.92 \%$ & $5.1(6.3)$ & $5.3(4.3)$ \\
All surgeries, excluding laparotomies ${ }^{\mathrm{a}}$ & 12 & 841 & $1.43 \%$ & $3.5(3.8)$ & $3.9(3.3)$ \\
Only cesarean deliveries & 11 & 4505 & $0.24 \%$ & $2.5(2.2)$ & $3.8(3.1)$ \\
\hline
\end{tabular}

The data is aggregated across study arms

${ }^{\mathrm{a}}$ This category includes hernia repair (4 deaths), prostatectomy (3 deaths), amputation ( 2 deaths), debridement ( 1 death), disarticulation ( 1 death), and hydrocelectomy (1 death) 
deaths occurred during emergent cesarean deliveries. Table 2 presents the POMR and average length of stay for each procedure category.

Laparotomy POMR ranged between 3.37 and $4.52 \%$ among the study cohorts. The non-obstetric, non-laparotomy POMR ranged between 0.99 and $2.44 \%$. Cesarean delivery mortality ranged between 0.17 and $0.36 \%$. Logistic regression demonstrated no statistically significant effect of the SS2020 intervention on POMR in any of the surgical procedure categories (Table 4). POMR rates were not risk adjusted due to missing data. However, urgency, ASA score, and wound class by procedure category are presented in Table 5 for reference.

\section{Discussion}

This study provides POMR estimates for various procedure categories in Tanzania's Lake Zone. The POMR for laparotomy (3.92\%) was highest compared to non-laparotomy POMR (1.43\%) and cesarean delivery POMR $(0.24 \%)$. This relative difference is likely due to the risk profile associated with laparotomies, but also highlights issues such as late presentation of disease. Despite the predictably higher rate of mortality of laparotomies compared to other surgeries in this study, it is lower than laparotomy estimates in other studies. A systematic review of LMICs showed a median POMR of $11.11 \%$ in laparotomies [15]. A study on laparotomy for gastric outlet obstruction in Tanzania found a $18.5 \%$ mortality rate [16]. The disparity with our laparotomy POMR estimates may be attributable to a relatively young population, referral of complex patients to tertiary referral hospitals, surgeon confidence in complex cases, or deaths occurring before presenting to the hospital. For example, the Million Death Study in India found that $71 \%$ of deaths due to acute abdominal conditions occurred at home [17]. Different health practices, cultural norms, healthcare referral structure, and infrastructure can contribute to death prior to arrival. The relatively high rate of clean wound class laparotomies in this study suggests that sicker patients may be dying before arrival or referred onto higher levels of care.

Cesarean delivery mortality $(0.24 \%)$ was significantly higher than the maternal mortality associated with spontaneous vaginal deliveries (SVD) $(0.03 \%)$. Patients who had cesarean delivery on average stayed longer in the hospital and had more complicated deliveries. Many SVD patients were discharged quickly, potentially resulting in underreported deaths. A higher POMR from cesarean delivery compared to SVD is consistent with international literature [18]. Compared to data from the ASOS study [6], the cesarean delivery mortality in this study was 2.2 times lower $(0.24 \%$ vs. $0.53 \%)$. In another study, it was 5.5 times lower when compared to other low-income countries $(0.24 \%$ vs. $1.32 \%)$ and 30 times higher when compared to high-income countries like the United Kingdom ( $0.24 \%$ vs. $0.008 \%$ ) [19]. The mortality from the current study is underestimated compared to other low-income estimates, as it did not capture intraoperative death, a common time of death in severe postpartum hemorrhage. The cesarean delivery mortality in this study more appropriately approximates emergent mortality, as there were zero deaths among elective cases. Transition to a higher proportion of elective cesarean deliveries, as is seen in high-income countries, could decrease mortality rates.

Patients died early in their course of stay, with $83 \%$ of patients dying in the first 7 days. This finding suggests that the cause of high mortality may reflect preoperative, intraoperative, or early postoperative factors. Preoperative factors include patient comorbidities, late presentation for surgery, and presurgical vital status. This finding is

Table 3 Demographic information and procedure volume for each study arm

\begin{tabular}{|c|c|c|c|c|}
\hline & \multicolumn{2}{|l|}{ Pre-intervention } & \multicolumn{2}{|l|}{ Post-intervention } \\
\hline & Control & Intervention & Control & Intervention \\
\hline \multicolumn{5}{|l|}{ Non-obstetric surgeries } \\
\hline Age, mean (sd) & $41.2(18.4)$ & $38.6(18)$ & $42.3(19.7)$ & $38.2(19.4)$ \\
\hline Percent female & $268 / 431(62.2 \%)$ & $260 / 412(63.1 \%)$ & $231 / 413(55.9 \%)$ & $232 / 382(60.7 \%)$ \\
\hline \# Laparotomies/total non-obstetric procedures & $227 / 431(52.7 \%)$ & $179 / 412(43.4 \%)$ & $202 / 413(48.9 \%)$ & $179 / 382(46.9 \%)$ \\
\hline \# Other procedures/total non-obstetric procedures & $204 / 431(47.3 \%)$ & $233 / 412(56.6 \%)$ & $211 / 413(51.1 \%)$ & $203 / 382(53.1 \%)$ \\
\hline \multicolumn{5}{|l|}{ Cesarean delivery } \\
\hline Age obstetric, mean (sd) & $25.4(6.3)$ & $24.9(6.2)$ & $25.9(6.5)$ & $25.5(6.5)$ \\
\hline \# Cesarean deliveries/total procedures $(\%)$ & $1112 / 1536^{\mathrm{a}}(72.4 \%)$ & $1123 / 1535^{\mathrm{a}}(73.2 \%)$ & $1089 / 1499^{\mathrm{a}}(72.6 \%)$ & $1208 / 1588^{\mathrm{a}}(76.1 \%)$ \\
\hline
\end{tabular}

${ }^{a}$ Denominator represents the total number of surgeries, including cesarean delivery and all other surgeries. Twelve procedures were classified into multiple categories. For example, in some cesarean deliveries, a laparotomy was also performed 
Table 4 Postoperative mortality rates for each study arm and procedure category

\begin{tabular}{|c|c|c|c|c|c|}
\hline & \multicolumn{2}{|c|}{ Pre-intervention } & \multicolumn{2}{|c|}{ Post-intervention } & \multirow[t]{2}{*}{$p$-value } \\
\hline & Control (\%) & Intervention $(\%)$ & Control (\%) & Intervention (\%) & \\
\hline \multicolumn{6}{|l|}{ Non-obstetric surgeries } \\
\hline Only laparotomies & 4 & 3.37 & 4.06 & 4.52 & 0.701 \\
\hline $\begin{array}{l}\text { All surgeries, excluding laparotomies/cesarean delivery } \\
\text { Obstetric }\end{array}$ & 0.99 & 1.30 & 2.44 & 0.99 & 0.283 \\
\hline Cesarean delivery & 0.27 & 0.36 & 0.19 & 0.17 & 0.718 \\
\hline
\end{tabular}

consistent with the ASOS study, in which $94.1 \%$ of mortalities occurred on day one [6]. With the majority of deaths occurring early during admission, the case can be made for reducing the follow-up time for POMR. While this has been shown to lead to underestimation of 30-day POMR [20], a shorter follow-up period would be more feasible for data monitoring in LMICs [21].

After 10 months of the SS2020 intervention, we did not observe a statistically significant effect on POMR. The study was underpowered to detect a change in pre-postintervention POMR between the control and intervention groups. Therefore, no conclusions can be drawn on the effectiveness of the intervention on reducing POMR. A larger sample size and a longer intervention period may detect an effect of the SS2020 intervention on POMR. Similar studies implementing the surgical safety checklist alone have demonstrated a significant reduction in POMR after just one year $[22,23]$. One intervention in Tanzania reduced POMR from 5.67 to $2.93 \%$ after implementation of a "Continuous Quality Improvement" approach including preoperative visits the day before surgery and appropriate medical management of patients after surgery [12]. The study had an intervention follow-up period of 1 and 2 years and a large sample contributing to their ability to detect changes.

Surgical care is becoming increasingly prioritized in global health efforts, as evident in the development of the National Surgical, Obstetric, and Anesthesia Plan (NSOAP) in Tanzania in 2018 [24] and the Southern African Development Community (SADC) [25] resolution calling for all SADC countries to create NSOAPs. NSOAP's are a response to the recognition that access to surgical care is a public health issue. They contain recommendations on monitoring surgical indicators to assess the current state of surgical capacity and monitor progress towards capacity building. This study provides data on POMR as one key surgical indicator required in the Tanzania NSOAP and can serve as a baseline for tracking surgical quality. The lack of power in this study highlights the need for large national datasets to benchmark and track changes in POMR. As surgery in LMICs are scaled, the quality of surgical care must be continuously monitored due to the potential for increasing POMR with increasing surgical volume [26]. This focus on surgical system quality alongside scaling of surgical delivery is essential for the eventual transformation of LMIC surgical systems [27].

\section{Limitations}

The number of deaths in this study is likely an underestimate as discharged or transferred patients were not followed after their hospital stay. Furthermore, as patients were only observed after surgery, deaths during surgery are also missing. Intra-operative deaths are an important measure of anesthesia safety and should be included in future investigations. This study focused on a small number of Tanzanian health facilities, with interventions implemented at only 10 facilities. The lack of power to detect changes in POMR resulting from the SS2020 intervention at control and intervention sites was anticipated, which is why a diverse array of indicators were used to evaluate the impact of the intervention. Future studies should consider larger samples to ensure that it is powered to detect changes in POMR.

\section{Conclusions}

This study provides estimates of in-hospital POMR for multiple procedure categories in the SS2020 study population in the Lake Zone of Tanzania. These results should be used as a baseline for measurement of future POMR as the Tanzania NSOAP continues to be implemented and evaluated. This study also showed no improvement in 
Table 5 Risk factor-specific mortality rates

\begin{tabular}{|c|c|c|c|c|}
\hline Surgery group & Risk factor & & Mortality rate & $\%$ Missing data ${ }^{a}$ \\
\hline \multirow[t]{11}{*}{ Laparotomy } & \multirow[t]{2}{*}{ Urgency } & Elective & $0.57 \%(1 / 174)$ & \multirow[t]{2}{*}{$39.1 \%$} \\
\hline & & Emergent & $3.77 \%(11 / 292)$ & \\
\hline & \multirow[t]{5}{*}{ ASA } & ASA 1 & $2.13 \%(4 / 188)$ & \multirow[t]{5}{*}{$45.1 \%$} \\
\hline & & ASA 2 & $4.31 \%(9 / 209)$ & \\
\hline & & ASA 3 & $0 \%(0 / 20)$ & \\
\hline & & ASA 4 & $0 \%(0 / 1)$ & \\
\hline & & ASA 5 & $0 \%(0 / 2)$ & \\
\hline & \multirow[t]{4}{*}{ Wound class } & Clean & $3.06 \%(9 / 294)$ & \multirow[t]{4}{*}{$35.8 \%$} \\
\hline & & Clean-Contaminated & $0.76 \%(1 / 132)$ & \\
\hline & & Contaminated & $4.35 \%(2 / 46)$ & \\
\hline & & Dirty & $5.26 \%(1 / 19)$ & \\
\hline \multirow[t]{11}{*}{ Surgeries (excluding laparotomy) } & \multirow[t]{2}{*}{ Urgency } & Elective & $2.03 \%(7 / 345)$ & \multirow[t]{2}{*}{$41.7 \%$} \\
\hline & & Emergent & $2.07 \%(3 / 145)$ & \\
\hline & \multirow[t]{5}{*}{ ASA } & ASA 1 & $0.9 \%(2 / 223)$ & \multirow[t]{5}{*}{$52.7 \%$} \\
\hline & & ASA 2 & $3.14 \%(5 / 159)$ & \\
\hline & & ASA 3 & $0 \%(0 / 7)$ & \\
\hline & & ASA 4 & $0 \%(0 / 1)$ & \\
\hline & & ASA 5 & $0 \%(0 / 8)$ & \\
\hline & \multirow[t]{4}{*}{ Wound class } & Clean & $1.47 \%(6 / 408)$ & \multirow[t]{4}{*}{$37.8 \%$} \\
\hline & & Clean-Contaminated & $1.25 \%(1 / 80)$ & \\
\hline & & Contaminated & $5 \%(1 / 20)$ & \\
\hline & & Dirty & $6.67 \%(1 / 15)$ & \\
\hline \multirow[t]{11}{*}{ Cesarean delivery } & \multirow[t]{2}{*}{ Urgency } & Elective & $0 \%(0 / 203)$ & \multirow[t]{2}{*}{$32.9 \%$} \\
\hline & & Emergent & $0.28 \%(8 / 2819)$ & \\
\hline & \multirow[t]{5}{*}{ ASA } & ASA 1 & N/A & \multirow[t]{5}{*}{$38.4 \%$} \\
\hline & & ASA 2 & $0.29 \%(8 / 2724)$ & \\
\hline & & ASA 3 & $0 \%(0 / 21)$ & \\
\hline & & ASA 4 & $0 \%(0 / 5)$ & \\
\hline & & ASA 5 & $0 \%(0 / 23)$ & \\
\hline & \multirow[t]{4}{*}{ Wound class } & Clean & N/A & \multirow[t]{4}{*}{$30.9 \%$} \\
\hline & & Clean-Contaminated & $0.26 \%(8 / 3077)$ & \\
\hline & & Contaminated & $11.54 \%(3 / 26)$ & \\
\hline & & Dirty & $0 \%(0 / 11)$ & \\
\hline
\end{tabular}

Risk factor data were taken from the OR logbook and matched to the surveillance tool for mortality. No risk adjustment was completed due to missing data

${ }^{a}$ Urgency, ASA, and wound class were recorded using the OR logbook, which is not directly linked to the surveillance tool for mortality. The two tools were combined using demographic data, procedure date, and location. Between 30.9 and $52.7 \%$ of data were missing due to incompatibility of tools

POMR immediately after implementation of the SS2020 intervention, possibly due to low power to detect change.

Acknowledgements We would like to express our sincere appreciation to the Ministry of Health, Community Development, Gender, Elderly and Children (MoHCDGEC), the President's Office-Regional Administration and Local Government in Tanzania (PORALG), the regional medical officers of Mara, Kagera, Shinyanga, Simiyu, and Geita regions and the frontline leadership and surgical team members in the study sites for their support of the study. We would like to thank the members of the surgical teams, facility leadership and the medical data collectors for their assistance with collecting high-quality data. We would like to acknowledge and thank all organizations and individuals who contributed to our early consultation on the design of the Safe Surgery 2020 multicomponent intervention as well as those organizations and individuals who have contributed to the larger Safe Surgery 2020 initiative including Assist International, Dalberg, Jhpiego, LifeBox, Project ECHO, SPECT, and WFSA.

\section{Compliance with ethical standards}

Conflict of interest The Program in Global Surgery and Social Change, Dalberg Advisors, Jhpiego, and Assist International receive funding from the GE Foundation for the Safe Surgery 2020 project. 
Shehnaz Alidina, Isabelle Citron, Erastus Maina, Gopal Menon, Cheri Reynolds, Chris Strader, John Varallo, Isabelle Citron, Adelina Mazhiqi, Meaghan Sydlowski, Taylor Wurdeman, and John G. Meara had financial support from GE Foundation for the submitted work. David Barash is employed by GE Foundation, which funded this work. John Varallo declares financial support from ELMA Philanthropies. Fabian Massaga reports receiving a Jhpiego FAA grant. John G. Meara declares support from the Kletjian Foundation and the Ronda Stryker and William Johnston Foundation. Ntuli Kapologwe and Sarah Maongezi have nothing to declare. The authors have had no financial relationships with any organizations that might have an interest in the submitted work in the previous 3 years and no other relationships or activities that could appear to have influenced the submitted work.

Ethics compliance Ethical approval was obtained from Tanzania's National Institute for Medical Research and Harvard Medical School. Verbal consent was obtained from all participants included in the study in Swahili. For patients under 18 years old, parental consent was obtained.

Human rights All procedures performed involving human participants were in accordance with the ethical standards of both Harvard Medical School (IRB17-1819) and Tanzania's National Institute for Medical Research (NIMR/HQ/R.8a/Vol. IX/2515) and with the 1964 Helsinki Declaration and its later amendments or comparable ethical standards.

Informed consent Informed consent was obtained from all individual participants included in the study.

Open Access This article is licensed under a Creative Commons Attribution 4.0 International License, which permits use, sharing, adaptation, distribution and reproduction in any medium or format, as long as you give appropriate credit to the original author(s) and the source, provide a link to the Creative Commons licence, and indicate if changes were made. The images or other third party material in this article are included in the article's Creative Commons licence, unless indicated otherwise in a credit line to the material. If material is not included in the article's Creative Commons licence and your intended use is not permitted by statutory regulation or exceeds the permitted use, you will need to obtain permission directly from the copyright holder. To view a copy of this licence, visit http://creativecommons. org/licenses/by/4.0/.

\section{References}

1. Shrime MG, Bickler SW, Alkire BC et al (2015) Global burden of surgical disease: an estimation from the provider perspective. Lancet Glob Health 3:S8-S9

2. Meara JG, Leather AJM, Hagander L et al (2015) Global surgery 2030: evidence and solutions for achieving health, welfare, and economic development. Lancet 386(9993):569-624

3. Bickler SN, Weiser TG, Kassebaum N et al (2015) Global burden of surgical conditions. In: Debas HT, Donkor P, Gawande A, Jamison DT, Kruk ME, Mock CN (eds) Essential surgery: disease control priorities, 3 Ed (vol 1). The World Bank, Washington (DC), p 25

4. Jauniaux E, Grobman WA (2016) Caesarean section: Introduction to the 'World's No. 1' surgical procedure. In: Sathya D (ed) Textbook of caesarean section. Oxford University Press, Oxford
5. Molina G, Weiser TG, Lipsitz SR et al (2015) Relationship between cesarean delivery rate and maternal and neonatal mortality. JAMA 314(21):2263-2270

6. Biccard BM, Madiba TE, Kluyts H-L et al (2018) Perioperative patient outcomes in the African surgical outcomes study: a 7-day prospective observational cohort study. Lancet LondEngl 391(10130):1589-1598

7. Bishop D, Dyer RA, Maswime S et al (2019) Maternal and neonatal outcomes after caesarean delivery in the African surgical outcomes study: a 7-day prospective observational cohort study. Lancet Glob 7(4):e513-e522

8. Sobhy S, Zamora J, Dharmarajah K et al (2016) Anaesthesiarelated maternal mortality in low-income and middle-income countries: a systematic review and meta-analysis. Lancet Glob Health 4(5):e320-327

9. Humphreys H (2009) Preventing surgical site infection. Where now? J Hosp Infect 73(4):316-22

10. Shrime MG, Bickler SW, Alkire BC et al (2015) Global burden of surgical disease: an estimation from the provider perspective. Lancet Glob Health 3(Suppl 2):S8-9

11. Marks I, Kamali P, Khan M (2016) Data for the sustainable development of surgical systems: a global collaboration. WDI surgical indicators data collection.

12. Bosse G, Abels W, Mtatifikolo F et al (2015) Perioperative care and the importance of continuous quality improvement-a controlled intervention study in three tanzanian hospitals. PLoS ONE 10(9):e0136156

13. World Health Organization (2015) 2015 Global Reference List of 100 Core Health Indicators. Available from: https://apps.who.int/ iris/bitstream/handle/10665/173589/WHO_HIS_HSI_2015.3_ eng.pdf;jsessionid=318D7199224B35A6032B246702B4F222?se quence $=1$

14. Alidina S, Kuchukhidze S, Menon G et al (2019) Effectiveness of a multicomponent safe surgery intervention on improving surgical quality in Tanzania's Lake Zone: protocol for a quasi-experimental study. BMJ Open 9(10):e031800

15. Ng-Kamstra JS, Arya S, Greenberg SLM et al (2018) Perioperative mortality rates in low-income and middle-income countries: a systematic review and meta-analysis. BMJ Glob Health 3(3): $\mathrm{e} 000810$

16. Jaka H, Mchembe MD, Rambau PF et al (2013) Gastric outlet obstruction at Bugando Medical Centre in Northwestern Tanzania: a prospective review of 184 cases. BMC Surg 13:41

17. Dare AJ, Ng-Kamstra JS, Patra J et al (2015) Deaths from acute abdominal conditions and geographical access to surgical care in India: a nationally representative spatial analysis. Lancet Glob Health 3(10):e646-653

18. Gebhardt GS, Fawcus S, Moodley J et al (2015) Maternal death and caesarean section in South Africa: results from the 2011-2013 saving mothers report of the national committee for confidential enquiries into maternal deaths. South Afr Med J 105(4):287-291

19. Sobhy S, Arroyo-Manzano D, Murugesu N et al (2019) Maternal and perinatal mortality and complications associated with caesarean section in low-income and middle-income countries: a systematic review and meta-analysis. Lancet 393(10184):1973-1982

20. Ariyaratnam R, Palmqvist CL, Hider P et al (2015) Toward a standard approach to measurement and reporting of perioperative mortality rate as a global indicator for surgery. Surgery 158(1):17-26

21. Guest GD, McLeod E, Perry WRG et al (2017) Collecting data for global surgical indicators: a collaborative approach in the Pacific region. BMJ Glob Health 2(4):e000376 
22. Haynes AB, Weiser TG, Berry WR et al (2009) A surgical safety checklist to reduce morbidity and mortality in a global population. N Engl J Med 360(5):491-499

23. Collaborative GlobalSurg (2019) Pooled analysis of WHO Surgical Safety Checklist use and mortality after emergency laparotomy. Br J Surg 106(2):e103-e112

24. Tanzania Ministry of Health, Community Development, Gender, Elderly and Children (2018) The United Republic of Tanzania National Surgical, Obstetric, and Anesthesia Plan (NSOAP) 2018-2025. Available from: https://www.pgssc.org/tanzaniansoap-launch

25. Southern African Development Community (2018) SADC Ministers of Health and Ministers Responsible for HIV and AIDS
Meet in Namibia 8th November 2018. Available from: https:// www.sadc.int/files/3315/4169/8409/Media_Statement_-_Joint_ Meeting_of_SADC_Ministers_of_Health_and_those_respon sible_for_HIV_and_AIDS_.pdf

26. Nepogodiev D, Martin J, Biccard B et al (2019) Global burden of postoperative death. Lancet LondEngl 393(10170):401

27. Kruk ME, Gage AD, Arsenault C et al (2018) High-quality health systems in the sustainable development goals era: time for a revolution. Lancet Glob Health 6(11):e1196-e1252

Publisher's Note Springer Nature remains neutral with regard to jurisdictional claims in published maps and institutional affiliations. 